\title{
Una formación participativa para el profesorado novel. El caso de la UPV/EHU
}

\section{Participatory training for novice teachers. The case of the University of the Basque Country}

\author{
Itziar Rekalde-Rodríguez ${ }^{1}$, Aitor Mendia-Urrutia ${ }^{2}$ \\ ${ }^{1}$ Universidad del País Vasco. itziar.rekalde@ehu.eus \\ ${ }^{2}$ Universidad del País Vasco. aitor.mendia@ehu.eus
}

Recibido: 6/04/2020

Aceptado: 26/10/2020

Copyright (C)

Facultad de CC. de la Educación y Deporte.

Universidad de Vigo

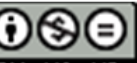

Dirección de contacto:

Itziar Rekalde-Rodríguez

Facultad de Educación, Filosofía y

Antropología UPV/EHU

Av./ Tolosa, 70

20018 Donostia-San Sebastián

\section{Resumen}

La Universidad del País Vasco (UPV/EHU) lanzó en 2017 un programa de formación del profesorado novel alineado con los ejes estratégicos de la institución.

El objetivo de este trabajo es describir y comprender qué ha significado para el profesorado novel la manera de abordar participativamente el programa formativo para su desarrollo académico. El enfoque metodológico es interpretativo articulado a través del estudio de caso. Como instrumentos de recogida de información se han utilizado entrevistas en profundidad, portfolios y análisis documental donde se recogen las actividades participativas desarrolladas durante la formación. El procedimiento de análisis ha consistido en la construcción de un sistema de categorías en el que emergen las voces de las personas participantes. Los resultados demuestran que la acción participativa a través de la que se ha abordado la formación ha sido significativa para el profesorado novel porque han podido co-construir su propio itinerario formativo, desarrollar la capacidad reflexiva, aprender con el grupo de iguales y transferir la formación. Se concluye que los diseños formativos en clave participativa pueden ayudar a impulsar la autonomía académica, la actitud reflexiva, y la investigación sobre sus prácticas docentes.

\section{Palabras clave}

Educación Superior, Formación de Profesorado Novel, Actividades Participativas, Estudio de Caso

\begin{abstract}
The University of the Basque Country launched a program to train novice teachers according to the institution's strategic lines in 2017.

The aim of this study is to describe and understand what the participatory approach to the training program has meant for novice teachers in their academic development. The methodological approach is interpretative and articulated through the case study. In this research has been used in-depth interviews, portfolios and documental analyze where the participatory activities developed along training are gathered. The procedure of analysis has consisted in the construction of a category system in which the voices of the participants emerge. The results show that the participatory action
\end{abstract}


through which the training has been approached has been significant for the novice teachers because they have been able to co-construct their own training itinerary, develop reflective capacity, learn with peer grouping and transfer what they have learnt. The study concludes that training designs in a participatory way can help to promote academic autonomy, reflective attitude, and researching about their teaching practices.

\section{Key Words}

Higher Education, Novice Teacher Training, Participatory Activities, Case Study

\section{INTRODUCCIÓN}

El Espacio Europeo de Educación Superior (EEES) ha generado un contexto lleno de cambios en el que la innovación y la formación del profesorado han estado muy presentes (Arandia y Rekalde, 2014; Gijón y Crisol, 2012; Knight, 2004; Madinabeitia y Fernández, 2017).

Se ha transitado de una formación puntual ofrecida a través de cursos y seminarios, a programas más amplios y complejos (Margalef y Álvarez, 2005), donde no sólo es necesario que el/la docente sepa de la materia, sino que sea competente en cómo enseñarla (López, 2011). Se puede afirmar que ahora no hay una clara división entre la formación profesional y la científico-académica (Álvarez et al., 2011), y que la formación basada en competencias es clave para el profesorado universitario (Mas, 2011; Zabalza, 2003). Profesorado que tiene que demostrar ser competente tanto en la investigación de su campo de conocimiento, en la enseñanza de su materia e investigación de ésta, y en la gestión universitaria (Caballero y Bolívar, 2015; Sánchez y Mayor, 2006; Tardif, 2004). Para ello es imprescindible el establecimiento de programas y procesos formativos sistemáticos y de calidad (Marentic, 2009); flexibles y adaptados a los contextos y a las necesidades de los y las participantes (Madinabeitia y Fernández, 2017), contemplando un itinerario profesional global de la persona participante (Dias, Gomes y Dias, 2005; Eirín, García y Montero, 2009), y con una visión comprensiva (Fernández et al., 2013).

\subsection{Los programas de formación para el profesorado novel en la educación superior}

La formación inicial del profesorado universitario se asienta en la idea básica de que el mejor modo de responder a los desafíos planteados desde la sociedad del conocimiento y del aprendizaje, es la profesionalización de la docencia universitaria (Fernández, 2008; Paricio, Fernández y Fernández, 2019), porque el profesorado novel está bien formado en cuanto a su ámbito de conocimiento, pero no así para la transferencia de éste al alumnado (Fernández, 2003; Herrera, Fernández, Caballero y Trujillo, 2011). La literatura recoge que los programas formativos dirigidos al profesorado novel deben: explicitar los motivos por los que el novel realiza la formación; generar compromiso del profesorado novel hacia la formación; ofrecer apoyo a lo largo del tiempo; acompañarles emocionalmente; vincular la formación a la realidad teniendo una visión de futuro; desarrollar el programa en su propio contexto; 
crear un clima propicio para el aprendizaje; y guiar la formación a la par que la reflexión (Bozu, 2010; Sánchez y Mayor, 2006).

La literatura nos recuerda que este colectivo requiere de una atención especial por hallarse en muchos casos inseguros, con falta de confianza e intentando establecer su propia identidad profesional, además de que las experiencias que se generan en esta inducción inicial tienen una gran influencia en la identidad profesional futura (Herreras, 2007).

\subsection{Una formación participativa para el profesorado novel}

Johnson, Adams, Estrada, y Freeman (2015) en su informe Horizon para la educación superior identifican el pensamiento complejo de la enseñanza como un desafío difícil de alcanzar a largo plazo y señalan que se necesitan modelos innovadores de educación para fomentar las habilidades críticas del siglo XXI. Estos nuevos enfoques pedagógicos deben desarrollar en quien aprende, en nuestro caso en el profesorado novel, competencias para participar en espacios de aprendizaje complejos que reflejen la diversidad del mundo real. De ahí que la formación se conciba como un proceso:

- en el que cada persona que aprende es un aprendiz activo y comprometido (Fernández et al., 2013);

- complejo de creación de sentido en el que cada persona que aprende aporta su propio contexto y tiene sus propias necesidades (Cormier, 2012);

- lleno de conexiones y significatividad para quien aprende (Díaz, 2003);

- dialéctico entre la práctica y la teoría que genera un saber propio, que permite transformarse en un sujeto activo capaz de generar nuevas hipótesis, supuestos y principios (Solé y Coll, 2000).

- amplio, flexible, continuo y caracterizado por una reflexión constante sobre la experiencia diaria (Popham, 2002);

- social en el que aprenden unas personas de otras; quien aprende lo hace, también, de su grupo de iguales (Boud y Lee, 2005), y

- en el que se confía en el conocimiento de los compañeros como un complemento importante a la labor del profesorado (programas interdisciplinares) (Gardner et al., 2012).

Para abordar este complejo entramado de procesos se proponen estrategias pedagógicas participativas en las que quien aprende se convierte en el eje del proceso formativo (Blanco, 2009; De Miguel, 2006; Martínez y Carrasco, 2006; Murillo, 2007), potenciando una intervención basada en el empoderamiento donde la gestión del conocimiento recae en quien aprende (Rekalde y Pérez-Sostoa, 2015), y siendo la reflexión la que conforma, articula y da sentido a los enfoques didácticos (Huber, 2008; Jay y Johnson, 2002). Trabajar con estrategias participativas, entendidas como prácticas de enseñanza-aprendizaje que involucran a las personas en su proceso de aprender, fortalece los mecanismos de comunicación mediante el encuentro interactivo entre las personas y provoca un aprendizaje más consistente y profundo (López, 2009; MartínezDomínguez et al., 2011).

Siendo conscientes de que evaluar un programa de formación consiste en centrar la atención en todos los elementos que han intervenido para poder tomar decisiones y valorar si el esfuerzo y la inversión merecen la pena (Pineda, 2000), en este trabajo el 
objeto de estudio no ha sido el programa en su totalidad, sino el impacto que la manera de abordar la formación ha tenido en las personas participantes para, en el futuro, seguir impulsando esta manera de hacer o repensar su idoneidad ${ }^{1}$. Dicho esto, el trabajo tiene por objetivo describir y comprender qué ha significado para el profesorado novel de la UPV/EHU la manera de abordar participativamente el programa formativo para el desarrollo académico.

\section{MÉTODO}

En coherencia con el marco teórico y el objetivo del estudio, se ha optado por un enfoque interpretativo para analizar percepciones, pensamientos y reflexiones respecto a las vivencias de las personas investigadas para que quien investiga comprenda sus puntos de vista (Janesick, 2000), puesto que el objeto de estudio -el impacto que la manera de abordar la formación ha tenido en el profesorado novel- es consecuencia de una situación histórica, cultural, económica y política en la que está inmersa la UPV/EHU (Blaxter, Hughes y Tight, 2008). Este enfoque se ha articulado con un abordaje de naturaleza cualitativa (Taylor y Bogdan, 1987) al objeto de comprender las voces recogidas en la formación (Grundy, 1991). La manera de abordar esta investigación ha sido a través del estudio de caso, dado que la literatura señala que constituye una de las estrategias más características del enfoque cualitativo (Bodgan y Biklen, 1982; Vázquez y Angulo, 2003), y lo encuentran particularmente apropiado para estudiar un caso o una situación con intensidad (Stake, 1998).

\subsection{Contexto}

En el contexto de la Universidad del País Vasco (UPV/EHU) se considera novel al profesorado adjunto, con una acreditación de ayudante-doctor y una experiencia docente de, al menos, dos cursos académicos con contrato a tiempo completo. En los últimos años ha ido aumentando el número de profesorado con este perfil debido, entre otros motivos, al relevo generacional que está viviendo la comunidad universitaria. Para dar respuesta se decidió intensificar la capacitación del profesorado novel.

El programa formativo se estructura a través de tres módulos a lo largo de 18 meses. En el primero se hace el diagnóstico y la proyectiva académica del novel a través de un diseño individualizado de formación denominado Plan de Aprendizaje Profesional (PAP) en el que se recogen metas de aprendizaje para el desarrollo de la competencia académica (docencia, investigación y gestión). El segundo y tercer módulo se centran en el desarrollo de los itinerarios individualizados del PAP (cursos, talleres, jornadas... formativas) $\mathrm{y}$ es en estos módulos cuando se inserta la estrategia del mentoring (profesorado universitario experimentado) para el acompañamiento del novel a lo largo del proceso formativo. El programa tiene un carácter voluntario u opcional, y al finalizar la formación, si la evaluación es positiva en los tres módulos, se les expide un certificado de formación de 10 ECTS que pueden utilizarlo como mérito ante las agencias externas de acreditación. 


\subsection{Participantes}

Son 18 docentes noveles matriculados en esta primera edición del programa quienes han participado en esta investigación. Respecto al perfil de los participantes, la edad promedio es de 36 años, predomina el sexo femenino, siendo once el número de mujeres, frente a siete varones. La participación por áreas de conocimiento es: Ciencias de la Salud (Medicina y Enfermería, Farmacia, Psicología...) con 6 participantes, Ciencias Sociales y Jurídicas (Derecho, Educación, Periodismo, Economía y Empresa...) y el área de las Ciencias Técnicas (Ingenierías y Arquitectura) con 4 participantes en cada una, Ciencias Experimentales (Química, Biología, Geología...) 3, y 1 en el área de Artes y Humanidades (Historia, Filología, Bellas Artes...).

En cuanto a las personas formadoras, han mantenido un doble rol como facilitadoras y guías del proceso formativo (Murillo, 2007), y como investigadoras del mismo (Cabo, 2006). Es decir, se ha asumido un doble rol a lo largo del proceso formativo.

\subsection{Instrumentos de investigación}

Los instrumentos han sido:

1) Análisis documental. Material generado a partir de las dinámicas participativas en clave reflexiva desplegadas durante la formación. Algunas de éstas han sido: El tren (T), en la que se ha inducido a que se elabore un relato en primera persona sobre el recorrido experimentado desde sus inicios como PDI (Personal Docente e Investigador) hasta el momento actual. Un relato de carácter exploratorio y reflexivo en el que se explicitan circunstancias y razones que permiten explicar y comprender la trayectoria profesional. A partir de las historias individuales se construye la devolución de la lectura transversal de los relatos, dando una visión de conjunto a las personas participantes para que, a partir de ahí, se pueda de-construir para construir las interpretaciones consensuadas entre investigadores/formadores y alumnado. El boomerang (B), en el que se les facilita un texto que el alumnado modifica de manera individual atendiendo a los dos movimientos del boomerang: 1) ¿Cómo reinterpreto este texto teniendo en cuenta el contexto del grado en el que imparto docencia? Y,2) ¿Qué actuaciones debo desplegar para llevarlo a la práctica? El ejercicio se contrasta a través del juego de espejo con dos compañeros/as al objeto de dialogar sobre lo reflexionado, compartir puntos de vista, clarificar ideas, incorporar aspectos significativos generados del diálogo... Otras dinámicas han sido el termómetro de valores, planteamiento de situaciones dilemáticas... (López, 2009; Martínez-Domínguez et al., 2011). La recogida de información a través de las dinámicas generadas en la formación es apropiada porque, al igual que en un grupo focal, las actividades y dinámicas participativas en grupo permiten a los individuos reaccionar y construir sobre las respuestas de otras personas del grupo (Stewart, Shamdasani y Rook, 2007). Esto a su vez facilita un modo etnográfico de investigación que, en este estudio, proporciona una visión del tipo de cultura de investigación en la que los participantes trabajan y a la que contribuyen en las dinámicas participativas como un microcosmos de sus culturas académicas más amplias (Denzin y Lincoln, 2005). Al participar de las dinámicas, el profesorado desarrolla una imagen de su entendimiento compartido (o sus diferencias de opinión) de lo que les aporta la formación y les reporta el grupo de iguales; mientras lo hacen, quienes asumen el rol de formadores/investigadores observan las relaciones que operan entre los 
miembros del grupo como una demostración de la cultura que está en proceso de ser descrita (Patton, 2002).

2) Entrevistas individuales en profundidad. Como señalan Jordi y Macías (2014) se ha tenido presente que al entrevistar, el saber escuchar es tanto o más importante que el saber preguntar. Partiendo de este supuesto, la entrevista en profundidad ha facilitado: una implicación interpersonal con la persona entrevistada y una aclaración de cuestiones o dudas que han ido surgiendo al hilo de la entrevista. Se animó a los participantes a seguir sus propias líneas de pensamiento en una conversación, de modo que las preguntas se convirtieran en una guía.

3) ePortfolios. Instrumento que constituye la síntesis del efecto que la formación tiene en el desarrollo de su identidad académica, las consecuencias que la actividad genera, las dudas planteadas, las reflexiones vertidas... En los ePortfolios se han planteado cuestiones reflexivas: "Al haber realizado esta actividad hay aspectos ahora de mi práctica profesional que antes no consideraba, y creo que sí debo tenerlo en cuenta porque..."; "Percibo cambios en mi forma de entender la docencia, en la forma de aprender de los estudiantes, en la forma de enseñar porque..."; "Tengo más claro lo que estoy haciendo y lo que necesito hacer para desarrollarme como profesional de la docencia e investigación..."; "Percibo un cambio, una evolución en mi forma de pensar y actuar como PDI a lo largo del programa formativo, porque...”. En definitiva, el profesorado novel ha ido recogiendo a lo largo de los meses el impacto que el programa ha tenido sobre ellos (Orbea-Aizpitarte, Cruz-Iglesias y Rekalde-Rodríguez, 2019).

\subsection{Análisis de datos y categorías de análisis}

Los datos generados a través de los diferentes instrumentos fueron sometidos a un proceso recursivo de análisis inductivo (Braun y Clarke, 2006) para establecer temas recurrentes (Flick, 2009; Patton, 2002). En este sentido el procedimiento analítico ha consistido en un proceso dinámico y sistemático que ha requerido identificar, seleccionar, categorizar, comparar e interpretar el contenido derivado de los instrumentos de investigación (Coffey y Atkinson, 2003), procediendo a un análisis de contenido temático de carácter flexible y abierto (Mucchielli, 2001). El resultado ha sido un sistema de categorías que emerge de las voces y reivindicaciones de las personas participantes (véase Tabla 1).

\begin{tabular}{cc}
\hline Categoría & Sub-categoría \\
\hline Co-construcción del itinerario formativo & Acreditación como motivación \\
\cline { 2 - 2 } & Sentimiento de autonomía \\
\cline { 2 - 2 } & Consciencia sobre su situación docente \\
\hline Desarrollo de la capacidad reflexiva & Valorización de la docencia \\
\cline { 2 - 2 } & Comprensioción a la actividad académica \\
\hline Aprendizajes generados en el grupo de iguales docente \\
\cline { 2 - 2 } & Crecimiento personal \\
\cline { 2 - 2 } & Diversidad de culturas académicas \\
\hline Transferencia de la formación & Grupo en redes sociales \\
\hline
\end{tabular}

Tabla 1. Sistema categorial emergente 


\subsection{Factores éticos}

El respeto por la libertad y autonomía de las personas participantes se ha defendido en todo momento informándoles desde el principio de la naturaleza del estudio y llegando a acuerdos sobre el uso de los datos, su análisis y divulgación (Blaxter, Hughes y Tight, 2008; Comité de Ética para las Investigaciones relacionadas con Seres Humanos, CEISH, 2019). Las voces y las aportaciones de estas personas han sido codificadas, siendo un elemento más para preservar la privacidad y el anonimato, y los valores como la dignidad e integridad. En la codificación el primer código equivale al instrumento: $\mathrm{E}=$ Entrevista/ $\mathrm{P}=\mathrm{ePortfolio} / \mathrm{DPG}=$ Material derivado de las dinámicas participativas, y el segundo código se refiere al PDI que lo ha expresado (PDI-1) o al tipo de DPG en el que se recoge la aportación ( $\mathrm{T}=$ Tren, $\mathrm{B}=$ Boomerang). De ahí que, por ejemplo, la siguiente codificación (E/PDI-5) equivale a datos procedentes de la entrevista en profundidad del PDI codificado con el número 5, o (DPG/T) equivale a datos procedentes de los documentos generados a partir de la dinámica participativa denominada El Tren.

\section{RESULTADOS}

Las aportaciones de las personas participantes ponen de relieve las cuestiones que han sido más significativas de la manera de abordar participativamente la formación. Se distinguen las siguientes: 1) la co-construcción del itinerario formativo individualizado; 2) el desarrollo de la capacidad reflexiva; 3) los aprendizajes generados en el grupo de iguales; y 4) la transferencia de la formación.

\section{1. ¿Qué ha significado para el profesorado novel la co-construcción de su itinerario formativo?}

A la hora de ir co-construyendo en el seno del grupo el itinerario formativo individualizado, los docentes rescatan que ha sido clave la motivación inicial por la que se apuntaron a la formación que fue conseguir la acreditación como profesorado permanente y que les ha acompañado durante todo el proceso. Asimismo, durante esta construcción participativa han sentido que han sido autónomos a la hora de decidir y apostar en la elección de las unidades formativas que incorporaban en su itinerario. Este ejercicio, además, les ha servido para resituarse como académicos, acomodándolo a su situación actual y contexto de trabajo, y ello les ha dado la posibilidad de dar valor a la actividad docente junto a otras que desempeñan, como puede ser la de investigación, cuyo valor nunca se pone en tela de juicio.

Todos y todas han coincidido en que se presentaron a la formación para clarificar y auto diseñar el itinerario que les permitiera acreditarse como docentes universitarios ante las agencias estatales. No obstante, se recogen otras expectativas que bien pueden agruparse en torno a recibir dirección y acompañamiento en el itinerario profesional, y aquellos cuya expectativa era en torno a recibir formación en general: "He sido siempre mucho de seguir formándome, y me parecía que este programa me daba una opción muy buena" (E/PDI-1); "Para un profesor novel es interesantísimo” (E/PDI-9); "Sí, y he conseguido más beneficios que los que inicialmente me planteaba, porque he podido hacer formaciones complementarias dentro del programa, sobre nuevas metodologías, y 
eso ha sido muy interesante; lo podía haber hecho fuera pero lo he hecho cuando estaba dentro. Y me alegro" (E/PDI-5). No obstante, las personas participantes comentan que hubieran necesitado antes en el tiempo esta formación para haber evitado tantos rompederos de cabeza como han tenido: "Ha sido muy valioso, pero en mi caso lo hubiera necesitado antes" (E/PDI-2).

Han agradecido este trabajo, sobre todo por la autonomía de la que han disfrutado a lo largo de la formación y particularmente en el diseño de su itinerario formativo: "Tú dibujabas y decidías cual era la orientación en la que te querías mover" (E/PDI-4). Destacan que el programa les ha permitido planificar, ordenar y priorizar los pasos que cada cual establecía para llevar a cabo el itinerario formativo: "Largo sí, pero eso garantiza el que te encarriles y consigas los objetivos que tú mismo como académico te has planteado" (E/PDI-3).

En este sentido, afirman que el programa da libertad para que cada cual analice sus necesidades formativas $\mathrm{y}$, tras esa indagación, dibujar el itinerario profesional y seguirlo, aprovechando de esta manera más y mejor el programa formativo: "Conocer lo que se espera de mí, dónde estoy ahora, y qué tengo que hacer para avanzar" (E/PDI-7); "Tener la estructura inicial en la cabeza del plan a seguir para mí ha sido fundamental" (DPG/B); "Me ha aportado una mirada con perspectiva" (DPG/B); "El desarrollo académico me pareció muy útil; dónde estoy, qué tengo que hacer, de gestión, de investigación..." (E/PDI-8); "El aprovechamiento mayor que he sacado al programa formativo ha sido ser más consciente de dónde estoy" (E/PDI-7).

La construcción compartida del itinerario formativo hace que sean conscientes de la situación que viven en el contexto universitario próximo y les resitúa ante el espacio que ocupan, que pueden ocupar y que quieren ocupar en la UPV/EHU: "Sinceramente me ha ayudado a adaptarme a la institución" (E/PDI-6); "Adaptarme a la UPV/EHU. Conocer qué soy en la UPV/EHU y conocer la propia institución” (E/PDI-11); "Conocer mejor el mundo en el que desarrollo mi trabajo" (DPG/B); "Ahora tengo un mayor y mejor conocimiento del funcionamiento de la UPV/EHU” (DPG/B); "Pues creo que mi visión ahora es amplia y profunda de la UPV/EHU y, de mi propia situación" (DPG/B); "Me ha ayudado a tomar consciencia y situación de los procedimientos universitarios, normativas, deberes, obligaciones, etc." (P/PDI-6); "Me parece una oportunidad increíble para tomar conciencia del lugar que ocupamos los docentes y sobre todo del que queremos ocupar. Está siendo un proceso duro pero altamente motivador y apasionante" (P/PDI-16).

En esa co-construcción del itinerario formativo los participantes reivindican que ha sido el ámbito de la docencia en el que más han trabajado y profundizado: "Lo asocio, sin duda, más con la docencia que con la investigación" (E/PDI-2). Han reflexionado sobre la docencia, sobre cómo diseñar una guía docente, sobre la importancia de las competencias, se han aproximado a las teorías pedagógicas y se han apropiado de diferentes estrategias didácticas: "He conocido formas nuevas de utilizar metodologías activas" (E/PDI-1); "En cuanto a metodología, voy introduciendo metodologías como el ABP, que antes no utilizaba" (E/PDI-7). Pero lo mejor es que lo aprendido lo han podido aplicar en el aula durante el período formativo: "Lo aprendido en clase lo intentaba aplicar con mi alumnado" (E/PDI-3); "Gracias al programa porque nos animaban a aplicar en clase lo que aprendíamos, y mucho de lo que he hecho ha sido muy bonito tanto para mí como para mi alumnado" (E/PDI-4); "He aumentado mi 
dedicación a la docencia, pero creo que he mejorado más de manera cualitativa (aun no habiendo salido todo perfecto),... también más asentada" (P/PDI-14). Sienten que es el ámbito en el que más han evolucionado porque, entre otras cuestiones, han sabido poner en valor el trabajo que supone la docencia universitaria y la investigación sobre su propia práctica.

El profesorado cree que se ha de hacer un gran trabajo de concienciación en los Departamentos porque en ciertos contextos las metodologías activas para el aprendizaje no son aún bien recibidas: "Hay que estar motivado, porque no es una cuestión de obligar a nadie" (E/PDI-4). Y es ahí, en esos Departamentos, donde la implementación se hace más costosa, porque: "Incorporar todas estas metodologías implica mucho trabajo y no se pueden hacer todas a la vez, hay que ir haciendo poco a poco (...). Para el profesorado demasiado trabajo, en la teoría estaba muy bien organizado, pero luego para corregir ha sido una locura" (E/PDI-8).

Las voces de los y las participantes subrayan que el cambio más significativo que el programa ha propiciado en ellas y ellos es en la revisión, re-conceptualización, y aplicación del sistema de evaluación del alumnado: "Me ha dado información, me ha dado herramientas y me ha hecho reflexionar" (E/PDI-7). Reduciendo el peso de la nota del examen final y dando valor a otras actividades que conviene insertar para el desarrollo de las competencias al hilo de evaluar en sintonía con las tareas realizadas a través de las metodologías activas.

\section{2. ¿Qué ha significado para el profesorado novel el desarrollo de la capacidad reflexiva?}

Si bien las personas participantes reconocen que el programa formativo les ha servido para planear su itinerario profesional, son conscientes de que la clave para ello han sido los procesos reflexivos que se han desplegado a través de las diferentes estructuras, dinámicas y agentes que han intervenido. Los participantes subrayan cómo las dinámicas reflexivas les han ayudado a dar sentido a los acontecimientos por los que transitaron en los inicios como docentes en la Universidad, además, consideran la reflexión como un ejercicio de crecimiento personal, y subrayan también que la reflexión les ha ayudado a comprender mejor la actividad docente universitaria actual.

Prueba de ello son las vivencias y acontecimientos que se relatan respecto a la trayectoria académica y personal que han experimentado durante el proceso formativo. Respecto a la capacidad reflexiva, la enfocan como una constante que les ha hecho resituarse en otros momentos de su trayectoria académica para, desde ahí, entresacar: ¿Qué les indujo a ser docentes universitarios? ¿Qué dificultades han atravesado? ¿Qué estrategias y/o recursos pusieron en marcha para superar las dificultades? ¿Qué apoyos se encontraron por el camino? Las diferentes dinámicas reflexivas propuestas han sido la antesala a la elaboración del itinerario formativo a corto y medio plazo.

De los datos se desprende que los participantes definen dos perfiles diferenciados de entrada a la carrera académica; uno, "aquel en el que se destaca que la docencia es algo que les ha atraído siempre, con cierta dimensión vocacional, que les gusta ser docentes, y cuyo desarrollo académico ha estado singularmente vertebrado por la docencia" (DPG/T) y, otro el de "investigador/a vocacional que llega a la docencia, y cuya experiencia en este terreno es agridulce, aunque se subraya lo positivo, y su desarrollo académico está singularmente vertebrado por la investigación" (DPG/T). Si bien 
inicialmente las motivaciones intrínsecas de los participantes venían para algunos de mejorar en el ámbito de la docencia para seguir en la faceta de investigador, otros, en cambio, reconocen haber podido disfrutar de y con la docencia. Las motivaciones extrínsecas halladas en el desarrollo han sido "la permanencia, promoción laboral, y apoyos personales (colegas...)" (DPG/T). Los participantes han reconocido que "los inicios en la Universidad fueron duros y estresantes en la docencia por el miedo escénico, por el tipo de contrato que en la mayoría de los casos era de sustituciones temporales, por todo el cúmulo de decisiones a tomar en un corto periodo de tiempo, y porque todo se hace en soledad" (DPG/T). Entre los elementos que sienten que les reforzaron en el inicio de su carrera académica fueron "el reconocimiento del estudiantado (de forma manifiesta o a través de las encuestas de evaluación docente); la ayuda de los colegas, el trabajo y esfuerzo constante, el considerar la docencia como un reto a afrontar $\mathrm{y}$, el afrontamiento continuo de los obstáculos en clave positiva (atrevimiento, pro-actividad)" (DPG/T).

Reconocen que el "tiempo reflexivo inicial" para el diseño de los itinerarios formativos, así como el "tiempo de reposo" que requiere la asimilación y confrontación de lo aprendido que se ha contemplado en las diferentes fases del programa: "Implica un proceso de aprendizaje y de entender tu carrera que necesita de reposo" (E/PDI-7); "Haría el primer módulo, incluso, más largo porque se han generado debates muy interesantes en grupo, pero claro... no hay tiempo" (DPG/B). No obstante, otros y otras participantes consideran que no se ha gestionado bien el tiempo: "Hay que cortar más nuestras intervenciones para poder gestionar mejor el tiempo de las sesiones" (DPG/B).

El programa les ha permitido descubrirse y comprender qué supone ser docenteinvestigador en la UPV/EHU: "Me ha ayudado a saber qué es ser docente" (E/PDI-3); "La enseñanza no sólo es dar la clase y ya; es más que eso, es profundizar en la metodología de enseñanza, hacer cosas innovadoras para ofrecerle al alumnado otro punto de vista" (E/PDI-4). El programa les ha ayudado a ser conscientes de lo que son, de dónde están y qué tienen y quieren hacer en el futuro: "La situación en la que estamos no es tan mala como la pintamos" (DPG/B); "La verdad es que si miro hacia atrás, el cursar estos módulos ha supuesto conocer y dar respuesta a un mar de dudas, además de aprender un montón. Ha sido el recabar conocimiento para poder tener un buen punto de partida de lo que quiero ser" (P/PDI-14); "La verdad es que la carga docente en el profesorado novel es casi siempre bastante elevada y sobre todo durante los primeros años por lo que resulta muy difícil dedicar el tiempo necesario a mejorar el grado de adquisición del resto de competencias" (P/PDI-5).

Las personas participantes hacen hincapié en la reflexión como la clave para el crecimiento profesional: "Para mí es muy valioso la auto reflexión sobre mi papel como docente" (DPG/B); "Una de las preguntas que nos hacían es, ¿Qué tipo de docente quieres ser?" (E/PDI-7); "El programa me ha hecho tener la obligación de reservarme tiempo para reflexionar" (DPG/B); "Tiempo, sin duda... un poco de aire para reflexionar, recapacitar y plantear las tareas que tengo por delante..." (P/PDI-1); "Pararme a pensar en los diferentes planos (competencias) del trabajo y en cómo me veo yo en cada uno de ellos" (DPG/B); "Obligación de reflexionar (yo siempre pospongo esto)" (DPG/B); "Me ha obligado a pararme a pensar la trayectoria que he recorrido hasta el momento en la UPV/EHU, recordando los momentos buenos y malos, las dificultades y las alegrías, pero sobre todo, he podido acordarme de las personas que 
han recorrido conmigo este camino, bien como compañeros o como referentes" (P/PDI$6)$.

\section{3. ¿Qué ha significado para el profesorado novel el grupo de iguales?}

Las personas participantes subrayan cómo la comunidad de profesores que se ha generado les ha servido para ir tejiendo aprendizajes emergentes derivados de: la diversidad de culturas académicas de las cuales procede el profesorado novel; las dudas, sugerencias y problemáticas abordadas en el grupo online (WhatsApp) al margen de los canales institucionales (moodle) y, la difusión y contraste de todo ello que se ha llevado a cabo a terceras personas que, aun no participando de la formación, se han podido nutrir de ella.

Respecto a las redes de relación que les ha facilitado el programa, las voces destacan con singularidad el beneficioso aporte que les ha brindado la posibilidad de relacionarse con profesorado de diferentes disciplinas y áreas de conocimiento. Les ha ayudado a relativizar su propia situación profesional, a empatizar con las demás personas, a no sentirse solos en el proceso de aprendizaje, a compartir otros puntos de vista diferentes y, valiéndose de esa diferencia, contrastar y dialogar a través de su propia experiencia: "Ves otros puntos de vista que te ayudan mucho" (E/PDI-2); "Para mí ese intercambio de impresiones con iguales ha sido mucho" (DPG/B); "Campos que, a priori, no se parecen al mío, pero que luego dan un montón de ideas" (E/PDI-1); "El ver las cosas desde distintas perspectivas, ha sido muy positivo" (DPG/B); "Lo de compartir experiencias entre nosotros lo valoro mucho" (DPG/B); "Para mí juntarme con personas de diferentes disciplinas que están en mi misma situación me tranquiliza mucho, para mí eso ha sido muy interesante" (E/PDI-10); "Cuando contaba las cosas me sentía comprendida por el grupo, y es que estamos todos igual" (DPG/B); "Conocí a gente de otras especialidades, otras facultades y te cuentan un montón de cosas que tú no sabes" (E/PDI-6); "Estoy muy contenta con el programa y me aporta muchas cosas, pero sobre todo sentir que hay un plan para la estabilización del PDI y sentirme comprendida por compañeros en la misma situación que yo" (P/PDI-9); "Hablar con personas de distintas áreas de conocimiento de la UPV/EHU que se encuentran en la misma situación que uno mismo ayuda a la hora de comentar las dudas e inquietudes que nos surgen" (P/PDI-6).

Es de destacar, asimismo, que el profesorado novel creó su grupo informal de WhatsApp, y todavía lo utilizan para resolver entre ellos dudas sobre la normativa universitaria, convocatorias de investigación, cursos de formación o decisiones de la UPV/EHU respecto a temas de docencia o situación laboral. Para ellos el generar este grupo les ha servido de gran apoyo: "Uff, se han escrito en el WhatsApp muchas dudas y todavía funciona" (E/PDI-3); "Para intercambiar información nos ha venido muy bien" (E/PDI-8); "Yo ahí comparto lo que me ocurre" (DPG/B). Ha sido una vía sencilla $\mathrm{y}$, al alcance de todos, para poder mantener una comunicación ágil y fluida entre los participantes en la formación.

Asimismo, las experiencias, conocimientos e intercambios generados en esta red multidisciplinar han dado pie a que los participantes lo compartieran a su vez con terceros en el contexto del Departamento, Facultad... Algunos lo han compartido de manera informal en los pasillos, a la hora del café... con compañeros del Departamento $y$, en otras ocasiones, en reuniones formales, por ejemplo de coordinación de asignatura: 
"Compartir el material no, pero sí las reflexiones y comentarios que se han hecho entre nosotros" (E/PDI-5); "He compartido la guía docente donde incluía muchas ideas de mis compañeros" (E/PDI-4). La formación ha tenido en algunos casos un impacto también en terceras personas.

\section{4. ¿Qué ha significado para el profesorado novel la transferencia de la formación?}

Las personas participantes vinculan la transferencia con la implementación de metodologías activas en su aula y con las dificultades que se han encontrado en su entorno al transferir lo aprendido en la formación.

Respecto a la transferencia de la formación adquirida en el programa, los participantes comentan situaciones muy diversas: algunos comentan que el alumnado ha visto con muy buenos ojos los cambios introducidos en la enseñanza, respondiendo positivamente y sintiendo, como docentes, que estaban motivados para el aprendizaje: "Jo, les generan muchas dudas las metodologías nuevas pero aprenden mucho y les gustan; les crean curiosidad" (E/PDI-5); "Son muy receptivos a los cambios, eso sí, siempre que no les suponga mucha carga de trabajo" (E/PDI-4). En este sentido de la carga de trabajo que las metodologías activas pueden suponer para el alumnado, la voz de la persona participante del Grado en Arquitectura desvelaba el rechazo de su alumnado hacia éstas: "Lo que he aprendido en la formación lo he aplicado y peor, el alumnado me dice que hacen mucho trabajo y me piden una y otra vez que les dé más clases magistrales" (E/PDI-10).

En cuanto a las resistencias por parte de los colegas, las respuestas al respecto han sido muy dispares; desde aquellos en cuyos contextos introducir cambios ha sido muy fácil: "Por parte del profesorado también muy bien, aunque otros muchos profesores no innoven su enseñanza" (E/PDI-6). "Sí, en general partidarios del cambio" (E/PDI-2). Donde señalan que los cambios han de ser introducidos de forma progresiva: "Los problemas surgen cuando lo quieres cambiar todo de repente; yo esto lo entiendo como algo que hay que ir cambiando a lo largo de los años" (E/PDI-4). No obstante, el profesorado novel se ha encontrado obstáculos para la transferencia, algunos puestos por parte de las personas coordinadoras de asignatura, grado...: "Cuando compartes docencia y propones nuevas actividades, son para todos, y a veces cuando son más mayores o así, pues no les hace mucha gracia y no siempre es posible"(E/PDI-8); "Le propuse al coordinador ciertas actividades, para que las implementásemos a modo de prueba, y me dijo que no"(E/PDI-3); "Sé que a parte de la gente con la que voy a trabajar les va a dar totalmente igual lo que vayamos a hacer, van a cumplir, pero no les va a gustar" (E/PDI-7). En líneas generales, como se reconoce en uno de los comentarios: "El programa me ha aportado una bofetada de realidad" (DPG/B); "El esfuerzo merece la pena" (DPG/B); "Sí, me ha ayudado un montón, el programa me ha encantado y se lo recomendaría a cualquiera" (E/PDI-6).

\section{DISCUSIÓN Y CONCLUSIONES}

Un programa de formación debe contemplar un itinerario profesional global de la persona participante (Dias, Gomes y Dias, 2005; Eirín, García y Montero, 2009), y los 
resultados nos han revelado que en la manera de abordar la formación los participantes subrayan la co-construcción del itinerario formativo individualizado como un elemento significativo con el que han dado sentido a lo aprendido desde sus propias necesidades actuales: docentes haciendo carrera universitaria (Cormier, 2012). Siendo la flexibilidad, la utilidad y la contextualización características de la co-construcción del itinerario formativo (Madinabeitia y Fernández, 2017; Zabalza, 2003).

Asimismo, en el sentido que apunta la literatura (Bozu, 2010; Fernández et al., 2013; Sánchez y Mayor, 2006), el programa abordado desde una clave participativa les ha ayudado a desarrollar la capacidad reflexiva, a dar sentido a lo ocurrido y a lo que acontece en la Universidad; a qué y por qué hacen lo que hacen en el aula y en el resto de los contextos universitarios en los que participan.

Respecto a los aprendizajes generados en el grupo de iguales han experimentado lo que significa (Boud y Lee, 2005) y la riqueza que aporta al grupo la diversidad, en este caso de culturas académicas, de las cuales procede el profesorado novel (Gardner et al., 2012). Asimismo, la confianza en el grupo de iguales al crear canales alternativos para la comunicación y el aprendizaje revelan una actitud activa y comprometida por parte del profesorado novel (Fernández et al., 2013).

En cuanto a la transferencia de la formación, los participantes han difundido y contrastado lo aprendido con terceras personas que, aun no participando de la formación se han podido nutrir de ella. El profesorado novel ha incorporado en sus prácticas docentes metodologías activas, trabajo colaborativo, prácticas reflexivas... con el alumnado (De Miguel, 2006; Martínez y Carrasco, 2006; Murillo, 2007), aunque a la hora de aplicar lo aprendido no haya estado libre de dificultades. De hecho, se puede evidenciar que las resistencias y dificultades al cambio son indicadores, en parte, del impacto de la formación.

Se concluye que los diseños formativos en clave participativa para el profesorado novel ayudan a:

- impulsar la autonomía en la construcción de un itinerario propio que aboga por el desarrollo académico del profesorado novel.

- ofrecer argumentos para que teoricen sobre su propia práctica, al objeto de comprenderla en profundidad y conseguir una clarificación conceptual de la docencia dando sentido y fundamento a su práctica docente.

- adoptar una actitud más crítica y reflexiva ante su propia práctica, que les lleva a un desarrollo profesional con sentido. Gracias, en parte, a la actividad reflexiva de las dinámicas participativas que les han permitido buscar e incorporar acciones que propician una mejora en el aprendizaje del alumnado.

- abrir el camino hacia la investigación sobre su propia práctica docente con el objetivo de mejorarla, valorarla y visibilizarla, haciendo aportaciones al campo de la enseñanza en su área científica.

- apostar estratégicamente para formar al profesorado que asumirá el testigo de liderar la institución universitaria.

\section{NOTAS}

${ }^{1}$ Quienes han escrito este artículo forman parte del grupo de investigación IKASGURA. Cambio educativo en la Universidad (Grupo reconocido por el Gobierno Vasco, nivel B-IT 13482019). Grupo comprometido y preocupado por abordar la investigación de los procesos de 
enseñanza-aprendizaje, la innovación, la identidad docente y el desarrollo curricular en la Educación Superior. Hacemos constar, además, que el autor Aitor Mendia-Urrutia disfruta actualmente de una beca pre doctoral en la convocatoria de contratación para la formación de personal investigador en la UPV/EHU 2019.

\section{BIBLIOGRAFÍA}

Álvarez, V., Romero, S., Gil, J., Rodríguez, J., Clares, J., Asensio, I., del Frago. R., García, B., García, M., González, B., Guardia, S., Ibarra, M., López, R., Rodríguez, G. y Salmerón, P. (2011). Necesidades de formación del profesorado universitario para la adaptación de su docencia al Espacio Europeo de Educación Superior. Revista Electrónica de Investigación y Evaluación Educativa, 17(1).

Arandia, M. y Rekalde, I. (2014). La formación de equipos de coordinación para el desarrollo curricular de los grados: el programa ehundu de la UPV/EHU). Revista de Docencia Universitaria, 12, 91-114.

Blanco, A. (2009). Desarrollo y evaluación de competencias en educación superior. Madrid: Narcea.

Blaxter, L., Hughes, C. y Tight, M. (2008). Cómo se investiga. Barcelona: Graó.

Bodgan, R.C. y Biklen, S.K. (1982). Qualitative research for education: an introduction to theory and methods. London: Allyn and Bacon.

Boud, D. y Lee, A. (2005). Peer learning as pedagogic discourse for research education. Studies in Higher Education, 30(5), 501-516.

Bozu, Z. (2010). El profesorado universitario novel: Estudio teórico de su proceso de inducción o socialización profesional. Revista Electrónica de Investigación y Docencia, 3, 55-72.

Braun, V. y Clarke, V. (2006) Using thematic analysis in psychology. Qualitative Research in Psychology, 3(2), 77-101.

Caballero, K. y Bolívar, A. (2015). El profesorado universitario como docente: hacia una identidad profesional que integre docencia e investigación. Revista de Docencia Universitaria, 13(1), 57-77.

Cabo, C.C. (2006). Pensar y pensarse: un deber para mejorar la práctica. Revista Iberoamericana de Educación, 39(2).

Coffey, A. y Atkinson, P. (2003). Encontrar sentido a los datos cualitativos. Medellín: Contus.

Comité de Ética para las Investigaciones relacionadas con Seres Humanos de la UPV/EHU CEISH (2019). https://www.ehu.eus/es/web/ceid/ceish/comite

Cormier, D. (2012). Embracing Uncertainty-Rhizomatic Learning in Formal Education. Disponible en: http://www.youtube.com/watch?v=VJIWyiLyBpQ

De Miguel, M. (2006). Métodos y modalidades de enseñanza en la educación superior. En M. De Miguel (Coord.). Metodologías de enseñanza y aprendizaje para el desarrollo de competencias. Madrid: Alianza Editorial.

Denzin, N.K. y Lincoln, Y.S. (Eds). (2005). The SAGE handbook of qualitative research. Thousand Oaks \& London: Sage.

Dias, P., Gomes, M.J. y Dias, A.A. (2005). Inservice training: e-learning as a new and promising approach. Interactive Educational Multimedia, 11, 89-103. 
Díaz, F. (2003). Cognición situada y estrategias para el aprendizaje significativo. Revista Electrónica de Investigación Educativa, 5(2).

Eirín, R., García, H.M. y Montero, L. (2009). Profesores principiantes e iniciación profesional. Estudio exploratorio. Revista de Currículum y Formación del profesorado, 13(1), 101-115.

Fernández, A. (2003). Formación pedagógica y desarrollo profesional de los profesores de universidad: Análisis de las diferentes estrategias. Revista de Educación, 331, 171-197.

Fernández, A. (2004). El portafolio docente como estrategia formativa y de desarrollo profesional. Educar, 33, 127-142.

Fernández, A. (2008). La formación inicial del profesorado universitario: el título de Especialista Universitario en Pedagogía Universitaria de la Universidad Politécnica de Valencia. Revista Interuniversitaria de Formación del Profesorado, 63(22), 161-187.

Fernández, G., Guisasola, G., Garmendia, M., Alkorta, I. y Madinabeitia, A. (2013). ¿Puede la formación tener efectos globales en la universidad? Desarrollo docente, metodologías activas y curriculum híbrido. Infancia y aprendizaje, 36(3), 387-400.

Flick, U. (2009). An introduction to qualitative research. Thousand Oaks \& London: Sage.

Gardner, S.K., Jansujwicz, J., Hutchins, K., Cline, B. y Levesque, V. (2012). Interdisciplinary doctoral student socialization. International Journal of Doctoral Studies, 7, 377-394.

Gijón, J. y Crisol, E. (2012). La internacionalización de la Educación Superior. El caso del Espacio Europeo de Educación Superior. Revista de Docencia Universitaria, 10(1), 389414.

Grundy, S. (1991). Producto y praxis del currículum. Madrid: Morata.

Herrera, L., Fernández, A.M., Caballero, K. y Trujillo, J.M. (2011). Competencias docentes del profesorado novel participante en un proyecto de mentorización. Implicaciones para el desarrollo profesional universitario. Revista de Currículum y Formación del Profesorado, 1(3), 213-241.

Herreras, E. (2007). El profesorado de educación superior. Formación para la excelencia. Revista de Educación Superior, 144(4), 139-141.

Huber, G.L. (2008). Aprendizaje activo y metodologías educativas. Revista de Educación, número extraordinario, 59-81.

Janesick, V.J. (2000). The choreography of qualitative research design: Minuets, improvisations, and cristalization. En N.K. Denzin e Y.S. Lincoln (Eds.). Handbook of Qualitative Research (pp. 379-399). London: Sage Publications.

Jay, J.K. y Johnson, K. L. (2002). Capturing complexity: a typology of reflective practice for teacher education. Teaching and Teacher Education, 18, 73-85.

Johnson, L., Adams, S., Estrada, V. y Freeman, A. (2015). NMC Horizon Report. Texas: The New Media Consortium.

Jordi, M. y Macías, B. (2014). La entrevista en profundidad como recurso pedagógico en los estudios de Trabajo Social y Educación Social. Potencialidades y retos para el aprendizaje teórico-práctico. Revista de Investigación en Educación, 12(1), 105-111.

Knight, P. (2004). El profesorado de Educación Superior. Formación para la excelencia. Madrid: Narcea.

López, F. (2009). Metodología participativa en la enseñanza universitaria. Madrid: Narcea. 
López, J.I. (2011). Un giro copernicano en la enseñanza universitaria: Formación por competencias. Revista de Educación, 356, 279-301.

Madinabeitia, A. y Fernández, I. (2017). El desarrollo docente del profesorado universitario: Análisis y sistematización del concepto. Teoría de la Educación, 29, 87-108.

Marentic, B. (2009). Improving the quality of teaching and learning in higher education through supporting professional development of teaching staff. Napredak, 150, 341-359.

Margalef, L. y Álvarez, J. M. (2005). La formación del profesorado universitario para la innovación en el marco de la integración del Espacio Europeo de Educación Superior. Revista de Educación, 337, 51-70.

Martínez, M. y Carrasco, S. (2006). Propuestas para el cambio docente en la universidad. Barcelona: Octaedro-ICE.

Martínez-Domínguez, I., Arandia, M., Alonso-Olea, M.J., Del Castillo, L., Rekalde, I. y Zarandona, E. (2011). Trabajar con metodologías participativas en la formación universitaria, todo un desafío. Investigación en la escuela, 75, 101-113.

Mas, O. (2011). El profesor universitario: sus competencias y formación. Revista de Currículum y Formación del Profesorado, 15(3), 196-211.

Mucchielli, A. (2001). Diccionario de métodos cualitativos en ciencias humanas y sociales. Madrid: Síntesis.

Murillo, P. (2007). Nuevas formas de trabajar en la clase: metodologías activas y colaborativas. En P. Murillo (coord.). El desarrollo de competencias docentes en la formación del profesorado (pp. 129-154). Madrid: MEC.

Orbea-Aizpitarte, G., Cruz-Iglesias, E. y Rekalde-Rodríguez, I. (2019). ¿Cómo puede el portafolio del alumnado ayudar a mejorar las prácticas docentes? Revista Digital de Investigación en Docencia Universitaria, 13(1), 17-37.

Paricio, J., Fernández, A. y Fernández, I. (Eds.). (2019). Cartografía de la buena docencia universitaria. Un marco para el desarrollo del profesorado basado en la investigación. Madrid: Narcea.

Patton, M. Q. (2002). Qualitative research and evaluation methods. Thousand Oaks \& London: Sage.

Pineda, P. (2000). Evaluación del impacto de la formación en las organizaciones. Educar, 27, 119-133.

Popham, W.J. (2002). Classroom Assessment. What Teachers Need to Know. Boston: Allyn and Bacon.

Rekalde, I. y Pérez-Sostoa, V. (2015). Construyendo tareas auténticas para el desarrollo de competencias profesionales en la educación superior: el uso del Role-Play en el Grado de Pedagogía. Revista d'Innovació Docent Universitària, 7, 81-96.

Sánchez, M. y Mayor, C. (2006). Los jóvenes profesores universitarios y su formación pedagógica. Claves y controversias. Revista de Educación, 339, 923-946.

Solé, I. y Coll, C. (2000). Los profesores y la concepción constructivista. En C. Coll, E. Martín, T. Mauri, M. Miras, J. Onrubia, I. Solé y A. Zabala. El constructivismo en el aula (pp. 413). Barcelona: Graó.

Stake, R.E. (1998). Investigación con estudios de casos. Madrid: Morata. 
Stewart, D.W., Shamdasani, P.N. y Rook, D.W. (2007). Focus groups: Theory and practice. Thousand Oaks \& London: Sage.

Tardif, M. (2004). Los saberes del docente y su desarrollo profesional. Madrid: Narcea.

Taylor, S.J. y Bogdan, R. (1987). Introducción a los métodos cualitativos de investigación. Barcelona: Paidos.

Tójar, J.C. (2001). Planificar la investigación educativa: Una propuesta integrada. Buenos Aires: Fundec.

Vázquez, R. y Angulo, F. (2003). Introducción a los estudios de casos. Los primeros contactos con la investigación etnográfica. Málaga: Aljibe.

Zabalza, M.A. (2003). Las competencias docentes del profesorado universitario: calidad y desarrollo profesional. Madrid: Narcea. 